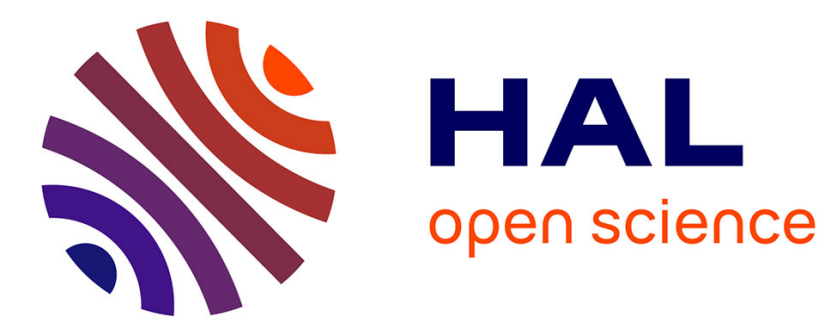

\title{
Capacity Gains of Some Frequency Reuse Schemes in OFDMA Networks
}

\author{
Thomas Bonald, Nidhi Hegde
}

\section{To cite this version:}

Thomas Bonald, Nidhi Hegde. Capacity Gains of Some Frequency Reuse Schemes in OFDMA Networks. Globecom, 2009, Honolulu, United States. 10.1109/GLOCOM.2009.5425225 . hal-01244793

\section{HAL Id: hal-01244793 \\ https://hal.science/hal-01244793}

Submitted on 16 Dec 2015

HAL is a multi-disciplinary open access archive for the deposit and dissemination of scientific research documents, whether they are published or not. The documents may come from teaching and research institutions in France or abroad, or from public or private research centers.
L'archive ouverte pluridisciplinaire HAL, est destinée au dépôt et à la diffusion de documents scientifiques de niveau recherche, publiés ou non, émanant des établissements d'enseignement et de recherche français ou étrangers, des laboratoires publics ou privés. 


\title{
Capacity gains of some frequency reuse schemes in OFDMA networks
}

\author{
Thomas Bonald Nidhi Hegde \\ Orange Labs, France \\ \{thomas.bonald, nidhi.hegde\}eorange-ftgroup.com
}

\begin{abstract}
The downlink capacity of cellular networks is known to be strongly limited by inter-cell interference. In order to mitigate this interference, a number of frequency reuse schemes have recently been proposed. In this paper, we compare the potential capacity gains of these schemes accounting for the random nature of traffic.
\end{abstract}

\section{INTRODUCTION}

Currently deployed cellular networks are known to be extremely sensitive to inter-cell interference. In HSDPA and CDMA-EVDO systems for instance, each base station transmits at full power when active, which strongly impacts the performance of neighbouring base stations. Avoiding or at least mitigating inter-cell interference is a key driver for the deployement of future systems based on Orthogonal Frequency Division Multiple Access (OFDMA).

OFDMA indeed provides the ability for each base station to selectively allocate frequency subbands and power to users depending on their location in the cell, according to some predefined frequency reuse pattern. A simple scheme consists for instance in dedicating a fraction of the frequency band to those users located "far" from the base station in terms of path loss; the other part of the frequency band is then used for "near" users with reduced power so as to limit the impact on neighbouring cells. By carefully allocating frequency and power in each cell, significant capacity gains are expected for the overall network.

A number of such frequency reuse schemes have recently been proposed and analysed, see [1]-[6] and references therein. It turns out, however, that the capacity gains are most often evaluated in static scenarios, without accounting for the random nature of traffic. In real systems, the number of active users varies over time with random requests for data transfers. It is this random environment that drives network dynamics and determines network capacity.

The objective of this paper is to evaluate the capacity gains of some practically interesting frequency reuse schemes, referred to as hard, soft and fractional reuse, under dynamic traffic conditions. Specifically, we consider a traffic model where randomly located users transfer files of random size at random times. The capacity of a cell is defined as the maximum traffic intensity it can sustain. We show that the soft reuse scheme is nearly optimal, with capacity gains ranging from $5 \%$ to $20 \%$ in typical network configurations.

Note that we do not include the effects of fast fading variations that may be exploited by opportunistic schedulers, see [7]. We also do not account for the dynamic coordination of base stations that may further increase network capacity [8]. The analysis of the gains due to the combination of these various schemes is left for future work.

The rest of the paper is organized as follows. We present the reuse schemes and the traffic model in the next section. Section III presents the network stability condition associated with each scheme, from which we deduce the capacity gain in section IV Section $\mathrm{V}$ concludes the paper.

\section{MODEL}

We focus on the downlink traffic in a typical cell of the network. We denote by $\mathcal{C}$ the serving zone of the considered cell and by $u \in \mathcal{C}$ the location of any user in the cell.

\section{A. Frequency reuse schemes}

A number of frequency reuse schemes have been proposed to mitigate inter-cell interference [1]-[6]. In the present paper, we focus on the following simple schemes:

Full reuse: The frequency band is fully used by each cell, independently of the location of users in the cell;

Hard reuse: The frequency band is divided into a fixed number of subbands that are allocated to the cells according to some predefined reuse pattern;

Soft reuse: The frequency band is divided into a fixed number of subbands; for each cell, one of these subbands is dedicated to "far" users, the rest is allocated to "near" users;

Fractional reuse: The frequency band is divided into a fixed number of subbands; a common subband is used by all base stations to serve "near" users; the other subbands, dedicated to "far" users, are allocated to the cells according to some predefined reuse pattern.

Note that the reuse factor is equal to 1 under both full and soft reuse since the whole frequency band is used by each base station, while it is less than 1 under hard reuse; under fractional reuse, it is equal to 1 for near users but is less than 1 for far users, hence the name. Examples of soft and fractional reuse are shown in Figure 1. The classification of a user as "near" or "far" is typically based on "distance" of the user to the base station, as estimated by the received power of the pilot signal. 
The interest of soft reuse compared to hard and fractional reuse lies in the full utilization of the frequency band by each base station. The potential reduction of inter-cell interference comes from the unequal power allocation on the frequency subbands dedicated to far and near users, respectively. In the soft reuse scheme of Figure 1 for instance, the center base station should typically transmit with reduced power on frequency subband 1 in order to limit its impact on neighbouring cells. As we shall see, both full and hard reuse may in fact be seen as limiting cases of soft reuse depending on the power allocation over subbands.

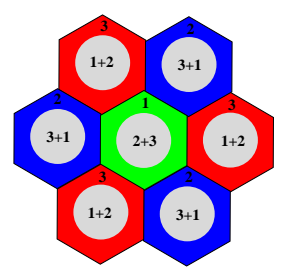

(a) Soft reuse with 3 frequency subbands

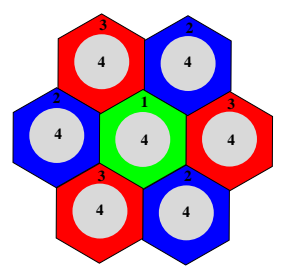

(b) Fractional reuse subbands with 4 frequency

Fig. 1. Some frequency reuse schemes in regular hexagonal networks.

Regarding soft and fractional reuse, we further consider two strategies:

Static scheme: There is a fixed threshold on the received power of the pilot signal that allows one to distinguish between far and near users;

Adaptive scheme: Far and near users are defined through the median received power of the pilot signal: half of users are considered as far, the others as near.

It is worth noting that, unlike the static scheme, the adaptive scheme doesn't require any parameter tuning. We shall see that the cell capacity achieved by the adaptive scheme in fact coincides with that achieved by the best static scheme (that is, for the optimal power threshold).

\section{B. Feasible throughput}

The throughput of any user located at $u \in \mathcal{C}$ depends on her/his radio conditions, and on the number of active users in the serving zone of the cell, $\mathcal{C}$. For each frequency reuse scheme, we refer to the feasible throughput of a user as her/his maximum throughput, obtained when there is no other active user in the cell. This is a function of the user location that characterizes the radio conditions on the serving zone $\mathcal{C}$; examples of such functions are given in Section IV] for various network configurations.

We assume a fair sharing of the frequency subbands among users (either in time or frequency, or both). We deduce the throughput of any user located at $u \in C$ :

Full reuse: Denoting by $R(u)$ the feasible throughput, the actual throughput is equal to $R(u) / n$ in the presence of $n$ active users in the cell.

Hard reuse: Denoting by $R^{\prime}(u)$ the feasible throughput, the actual throughput is equal to $R^{\prime}(u) / n$ in the presence of $n$ active users in the cell.
Soft or fractional reuse: Denoting by $R_{1}(u)\left(\right.$ resp. $R_{2}(u)$ ) the feasible throughput of a near user (resp. far user), the actual throughput of a near user (resp. far user) is equal to $R_{1}(u) / n_{1}$ (resp. $\left.R_{2}(u) / n_{2}\right)$ in the presence of $n_{1}$ active near users (resp. $n_{2}$ active far users).

The assumption of fair sharing simplifies the description of the underlying queueing system but is not critical for the stability results presented in Section III and the corresponding capacity gains derived in Section IV

\section{Traffic scenario}

We assume that users transfer digital documents like Web pages, audio and video files at random times. Each data transfer, referred to as a flow, adapts its rate to the user throughput available on the considered radio downlink. Thus the duration of any given flow depends not only on its size (in bits) but on the location of the user, which is assumed fixed during the data transfer, and on the number of active users in the cell. Under full reuse for instance, a flow of size $s$ started by a user located at $u \in \mathcal{C}$ at time $a$ is completed at time $b$ such that $\int_{a}^{b} \frac{R(u)}{N(t)} d t=s$, where $N(t)$ denotes the number of active users in the cell at time $t$.

Flows are assumed to be generated at rate $\lambda$. We denote by $\delta$ the traffic density in the cell, with $\int_{\mathcal{C}} \delta(u) d u=1$. Thus for all $u \in \mathcal{C}$, new flows arrive at rate $\lambda \delta(u) d u$ in any infinitesimal region of area $d u$ around $u$. Flows generated by users located at $u \in \mathcal{C}$ have i.i.d. sizes of mean $\sigma$ bits. We refer to the traffic intensity of the cell as the average volume generated by users per time unit (in bit/s). This is the product $\rho$ of the flow arrival rate and the mean flow size:

$$
\rho=\lambda \sigma .
$$

\section{Markovian description}

Let $N(t)$ be the number of active users at time $t$. The cell state is defined by the $N(t)$-dimensional vector $X(t)$ giving the location of each active user at time $t$.

In the specific case of Poisson arrivals and exponential flow sizes, $X(t)$ is a Markov process. Although these statistical characteristics are not representative of real traffic, they simplify the subsequent stability analysis. For all $n \geq 1$ and $x \in \mathcal{C}^{n}$, the transition rate from state $x$ corresponding to the generation of a new flow by a user located in a region of surface $d u$ around $u \in \mathcal{C}$ is equal to $\lambda \delta(u) d u$.

The transition rate from state $x$ corresponding to the completion of a flow by a user located at $u \in \mathcal{C}$ depends on the frequency reuse scheme. It is equal to $R(u) /(n \sigma)$ and $R^{\prime}(u) /(n \sigma)$ under full and hard reuse, respectively. Under soft or fractional reuse, it is equal to $R_{1}(u) /\left(n_{1} \sigma\right)$ if $u \in \mathcal{C}_{1}$, where $\mathcal{C}_{1}$ denotes the region of near users and $n_{1}$ is the number of near users in state $x$, and to $R_{2}(u) /\left(n_{2} \sigma\right)$ otherwise (i.e. $u \in \mathcal{C}_{1}$ ), where $\mathcal{C}_{2}$ denotes the region of near users and $n_{2}$ is the number of near users in state $x$. The regions $\mathcal{C}_{1}, \mathcal{C}_{2}$ are fixed under the static scheme and are functions of the state $x$ under the adaptive scheme. 


\section{Stability RESUlTS}

In this section, we are interested in the stability condition of the Markov process described in \$II-D We deduce the cell capacity, defined as the maximum traffic intensity compatible with stability, associated with each frequency reuse scheme.

\section{A. Full and hard reuse}

Under both full and hard reuse, the number of active users in the cell behaves as the number of customers in a single-server queue. Customers arrive according to a Poisson process of intensity $\lambda$. Under full reuse, they require exponential service times with mean $\int_{\mathcal{C}} \frac{\sigma}{R(u)} \delta(u) d u$. This follows from the fact that a flow generated by a user located at $u \in \mathcal{C}$ requires on average $\sigma / R(u)$ service time units at the base station. Such flows are generated with density $\delta(u)$. The queue is stable if its load is strictly less than 1 , that is

$$
\lambda \int_{\mathcal{C}} \frac{\sigma}{R(u)} \delta(u) d u<1 .
$$

We deduce the cell capacity, defined as the maximum traffic intensity $\rho=\lambda \sigma$ compatible with stability:

$$
C=\left(\int_{\mathcal{C}} \frac{\delta(u)}{R(u)} d u\right)^{-1} .
$$

This corresponds to the harmonic mean of the feasible throughput, weighted by the traffic density. The cell capacity under hard reuse, with the feasible throughput $R(u)$ replaced by $R^{\prime}(u)$.

\section{B. Static soft or fractional reuse}

Under static soft or fractional reuse, the numbers of active users in cell regions $\mathcal{C}_{1}, \mathcal{C}_{2}$ behave as two independent singleserver queues. Denote by $\delta_{1}$ and $\delta_{2}$ the fractions of flow arrivals in these two regions:

$$
\delta_{1}=\int_{\mathcal{C}_{1}} \delta(u) d u, \quad \delta_{2}=\int_{\mathcal{C}_{2}} \delta(u) d u .
$$

Customers arrive in the two queues according to independent Poisson processes of intensities $\delta_{1} \lambda, \delta_{2} \lambda$ and require exponential service times with respective means:

$$
\frac{1}{\delta_{1}} \int_{\mathcal{C}_{1}} \frac{\sigma}{R_{1}(u)} \delta(u) d u, \quad \frac{1}{\delta_{2}} \int_{\mathcal{C}_{2}} \frac{\sigma}{R_{2}(u)} \delta(u) d u .
$$

The two queues are stable if their load is strictly less than 1 , that is,

$$
\lambda \int_{\mathcal{C}_{1}} \frac{\sigma}{R_{1}(u)} \delta(u) d u<1 \quad \text { and } \quad \lambda \int_{\mathcal{C}_{2}} \frac{\sigma}{R_{2}(u)} \delta(u) d u<1 .
$$

We deduce the cell capacity $C=\min \left(C_{1}, C_{2}\right)$, with

$$
C_{1}=\left(\int_{\mathcal{C}_{1}} \frac{\delta(u)}{R_{1}(u)} d u\right)^{-1} \quad \text { and } \quad C_{2}=\left(\int_{\mathcal{C}_{2}} \frac{\delta(u)}{R_{2}(u)} d u\right)^{-1} \text {. }
$$

The partitioning of the cell into two regions dedicated to near and far users is optimal in terms of capacity when $C_{1}=C_{2}$. We denote by $\mathcal{C}_{1}^{\star}$ and $\mathcal{C}_{2}^{\star}$ the corresponding cell regions (which are uniquely defined) and by $C^{\star}$ the resulting cell capacity.

\section{Adaptive soft or fractional reuse}

Under adaptive soft or fractional reuse, the $\lceil n / 2\rceil$ nearest users are considered as "near" while the $\lfloor n / 2\rfloor$ farthest users are considered as "far" (there is no predefined power threshold). It can be proved using standard fluid limit technics [9] that the Markov process $X(t)$ is then stable if $\rho<C^{\star}$. Thus the corresponding cell capacity is that achieved by the best static scheme, $C^{\star}$.

\section{CAPACITY GAINS}

This section is devoted to the application of previous theoretical results to the evaluation of the capacity gains achievable by the considered frequency reuse schemes in various network configurations.

\section{A. Radio model}

We use the Shannon formula for the Gaussian channel as a model of the physical layer. Note that the model and analysis remain valid for the feasible throughput being any arbitrary function of the SINR. Specifically, the feasible throughput is assumed to be equal to

$$
W \log _{2}(1+\eta)
$$

where $W$ is the frequency bandwidth and $\eta$ is the SINR, averaged over fast fading variations. This is the maximum throughput that can be achieved by any modulation and coding scheme, in the absence of advanced radio techniques like cell coordination or opportunistic scheduling. Since the throughput of real systems is approximately logarithmic in the SINR, see [10] for instance, the considered model does not only give an upper bound on the capacity of each scheme but is expected to provide accurate results on the capacity gain of each scheme, compared to full frequency reuse.

We first neglect the thermal noise in order to magnify the impact of inter-cell interference, which is representative of dense networks in an urban environment; the impact of thermal noise is considered in $\$ \mathrm{IV}-\mathrm{F}$ Locating the reference base station at $v=0$ and denoting by $\mathcal{B}$ the set of locations of other base stations, the signal-to-interference ratio at location $u \in \mathcal{C}$ is given by:

$$
\eta(u)=\frac{|u|^{-\alpha}}{\sum_{v \in \mathcal{B}}|u-v|^{-\alpha}},
$$

where $\alpha$ is the path loss exponent.

In case of directional antennas, we add a linear angular path loss (in $\mathrm{dB}$ ) given by $h(\theta)=\frac{|\theta|}{\theta_{0}} h_{0}$, where $\theta$ is the angle from the beam axis to the user location, $\theta_{0}$ is the angle from the beam axis to the sector edge, and $h_{0}$ is the angular path loss at sector edge (in $\mathrm{dB}$ ). Unless otherwise specified, we take the standard values $\alpha=3.5$ and $h_{0}=9 \mathrm{~dB}[11]$.

\section{B. Network topology}

We consider two types of networks: 1-dimensional, linear networks and 2-dimensional, hexagonal networks, illustrated by Figures 2 and 3 . Sectorized versions of these networks contain 2 and 3 sectors per base station, respectively. 

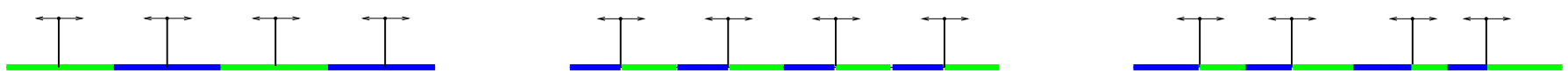

Fig. 2. Linear networks: unsectorized, regular network (left), sectorized, regular network (center), sectorized, random network (right).

By convention, cells have unit area in unsectorized, regular networks. The random versions of sectorized networks are obtained by distributing each base station uniformly at random in a disk of area $\varepsilon$ centered at its original location. For hexagonal networks, the direction of each antenna is also uniformly distributed in a cone of angle $\varphi$ centered on the original beam axis. For the numerical applications, we take $\varepsilon=1 / 4$ and $\varphi=\pi / 3$ and average the results over 100 network instances. Figures 2-(c) and 3-(c) show typical instances of such networks. Observe that the sectorized, random hexagonal network is the most representative of real networks in urban environment. In all cases, we assume a uniform traffic density in the network.

\section{Full and hard reuse}

The total frequency band has unit width by convention. Thus the feasible throughput at a location $u \in \mathcal{C}$ under full reuse is given by:

$$
R(u)=\log _{2}(1+\eta(u)),
$$

where the signal-to-interference ratio $\eta(u)$ is given by 3 .

Under hard reuse, we consider a reuse factor of $1 / K$, with $K=2$ for linear networks and $K=3$ for hexagonal networks. For linear networks, the two frequency bands are alternately allocated to cells, as illustrated by Figure 2 For hexagonal networks, we use the regular pattern of Figure 3 -(a) for omnidirectional antennas; for directional antennas, all antennas having the same direction (or the same original direction for the random version of the network) use the same frequency subband. The feasible throughput at location $u \in \mathcal{C}$ is then given by:

$$
R^{\prime}(u)=\frac{1}{K} \log _{2}\left(1+\eta^{\prime}(u)\right)
$$

Note that the corresponding signal-to-interference ratio $\eta^{\prime}(u)$ is larger than that obtained under full reuse, $\eta(u)$, due to the reduction of the number of interfering antennas. As mentioned in Section $\Pi$ and shown below, both the full and hard reuse schemes turn out to be limiting cases of the soft reuse scheme.

\section{Soft reuse}

a) Linear networks: For linear networks, we use two frequency bands of identical width in each cell. We denote by $\kappa=P_{1} / P_{2}$ the ratio of the power allocated to near users to the power allocated to far users, expressed in $\mathrm{dB}$. Thus the feasible throughputs of near and far users at location $u \in \mathcal{C}$ are respectively given by:

$R_{1}(u)=\frac{1}{2} \log _{2}\left(1+\eta_{1}(u)\right)$ and $R_{2}(u)=\frac{1}{2} \log _{2}\left(1+\eta_{2}(u)\right)$,

where the signal-to-interference ratios $\eta_{1}$ and $\eta_{2}$ depend on the factor of power asymmetry $\kappa$.
The corresponding cell capacity $C$ is shown as a function of $\kappa$ in Figure 4 This is achieved either by the static scheme with an optimal choice of the received power threshold or, in view of Theorem 1 , by the adaptive scheme. Note that the limiting cases $\kappa \rightarrow-\infty$ and $\kappa \rightarrow+\infty$ (in $\mathrm{dB}$ ) correspond to the hard reuse scheme: letting $P_{1}$ tend to 0 shrinks the cell region $\mathcal{C}_{1}^{\star}$ dedicated to near users, so that all users tend to be considered as far; similarly, letting $P_{2}$ tend to 0 shrinks the cell region $\mathcal{C}_{2}^{\star}$ dedicated to far users, so that all users tend to be considered as near.

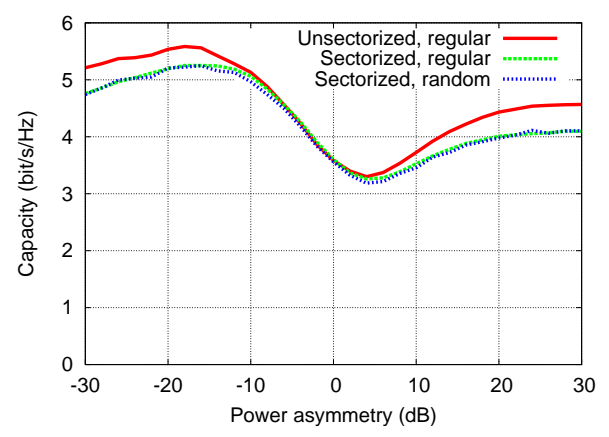

Fig. 4. Cell capacity in linear networks under soft frequency reuse.

Let us now show that the cell capacity under symmetric power allocation, $\kappa=0 \mathrm{~dB}$, coincides with that obtained under the full reuse scheme. Since $\eta_{1}(u)=\eta_{2}(u)=\eta(u)$ in this case, we have $R_{1}(u)=R_{2}(u)=R(u) / 2$ in view of (4). Now, using the fact that $C=C_{1}=C_{2}$, it follows from (2) that:

$$
\begin{aligned}
C & =\left(\frac{1}{2}\left(\frac{1}{C_{1}}+\frac{1}{C_{2}}\right)\right)^{-1} \\
& =\left(\frac{1}{2}\left(\int_{\mathcal{C}_{1}^{\star}} \frac{\delta(u)}{R_{1}(u)} d u+\int_{\mathcal{C}_{2}^{\star}} \frac{\delta(u)}{R_{2}(u)} d u\right)\right)^{-1} \\
& =\left(\int_{\mathcal{C}} \frac{\delta(u)}{R(u)} d u\right)^{-1},
\end{aligned}
$$

which corresponds to the cell capacity under full reuse, (1).

The capacity gains of the hard and soft reuse schemes compared to the full reuse scheme are shown in Table I(a) Under soft reuse, the capacity gain is calculated for the optimal value of $\kappa$ (around $-15 \mathrm{~dB}$ ).

b) Hexagonal networks: For hexagonal networks, we consider the scheme pictured in Figure 1-(a). The frequency band is divided into 3 subbands of identical width. The ratio of the power allocated to near and far users is denoted by $\kappa$ as above. The feasible throughputs of near and far users at location $u \in \mathcal{C}$ are respectively given by:

$R_{1}(u)=\frac{2}{3} \log _{2}\left(1+\eta_{1}(u)\right) \quad$ and $\quad R_{2}(u)=\frac{1}{3} \log _{2}\left(1+\eta_{2}(u)\right)$, 

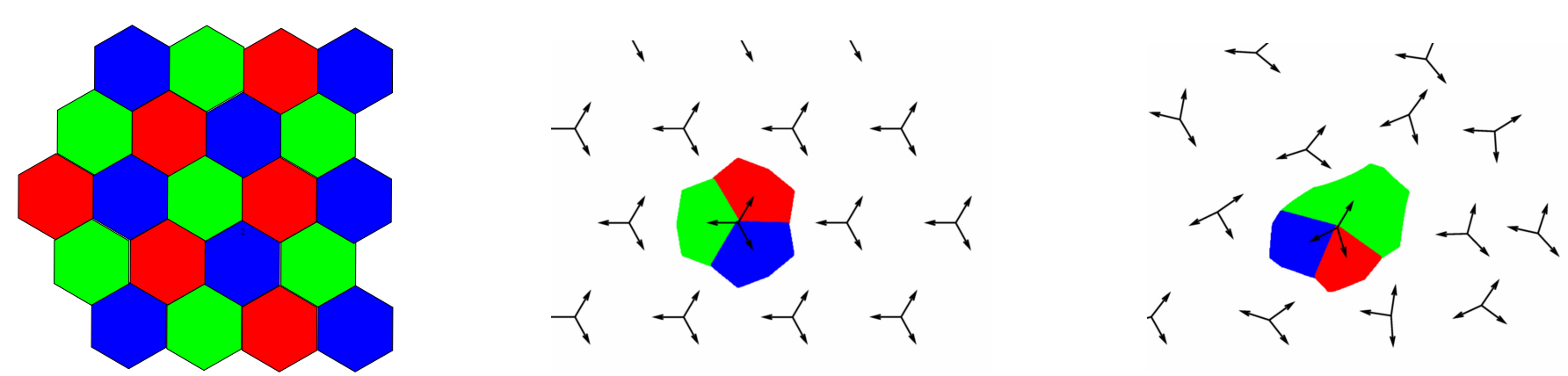

Fig. 3. Hexagonal networks: unsectorized, regular network (left), sectorized, regular network (center), sectorized, random network (right).

TABLE I

CAPACITY GAINS

(a) Linear networks

\begin{tabular}{|c|c|c|}
\hline Type of network & Hard reuse & Soft reuse \\
\hline Unsectorized, regular & $+27 \%$ & $+55 \%$ \\
Sectorized, regular & $+14 \%$ & $+44 \%$ \\
Sectorized, random & $+14 \%$ & $+44 \%$ \\
\hline
\end{tabular}

(b) Hexagonal networks

\begin{tabular}{|c|c|c|}
\hline Type of network & Hard reuse & Soft reuse \\
\hline Unsectorized, regular & $+7 \%$ & $+21 \%$ \\
Sectorized, regular & $0 \%$ & $+12 \%$ \\
Sectorized, random & $-5 \%$ & $+7 \%$ \\
\hline
\end{tabular}

where the signal-to-interference ratios $\eta_{1}$ and $\eta_{2}$ depend on the factor of power asymmetry $\kappa$.

The cell capacity is given in Figure 5 as a function of $\kappa$. The limiting cases $\kappa \rightarrow-\infty$ and $\kappa \rightarrow+\infty$ (in $\mathrm{dB}$ ) still correspond to the hard reuse scheme, but for different reuse factors: $1 / 3$ in the former case (since all users tend to be considered as far), $2 / 3$ in the latter case (since all users tend to be considered as near). The cell capacity of the full reuse scheme now corresponds to the case $\kappa=3 \mathrm{~dB}$ : the cell region $\mathcal{C}_{1}^{\star}$ dedicated to near users can then be divided into two subregions with the same frequency bandwidth and power as those of the cell region $\mathcal{C}_{2}^{\star}$, and it may be easily verified as above that the cell capacity is given by (1).

The corresponding capacity gains are shown in Table I(b) Note that the hard reuse scheme does not increase the capacity of sectorized networks. It actually decreases capacity in the most realistic case of sectorized, random networks.

\section{E. Fractional reuse}

We now consider the fractional reuse scheme illustrated by Figure 1. (b) where a common frequency subband is dedicated to near users. We consider the three types of hexagonal networks shown in Figure 3 There are three frequency bands of identical width dedicated to far users. These bands, numbered $1,2,3$, are allocated to cells as in the hard reuse scheme, cf. $\$ \mathrm{IV}-\mathrm{C}$. The subband dedicated to near users, numbered 4, has width $\xi$ (as a fraction of the total bandwidth). The same power is used for each subband.

The resulting cell capacity is shown in Figure 5 as a function of the frequency bandwidth $\xi$ dedicated to near users. Note that
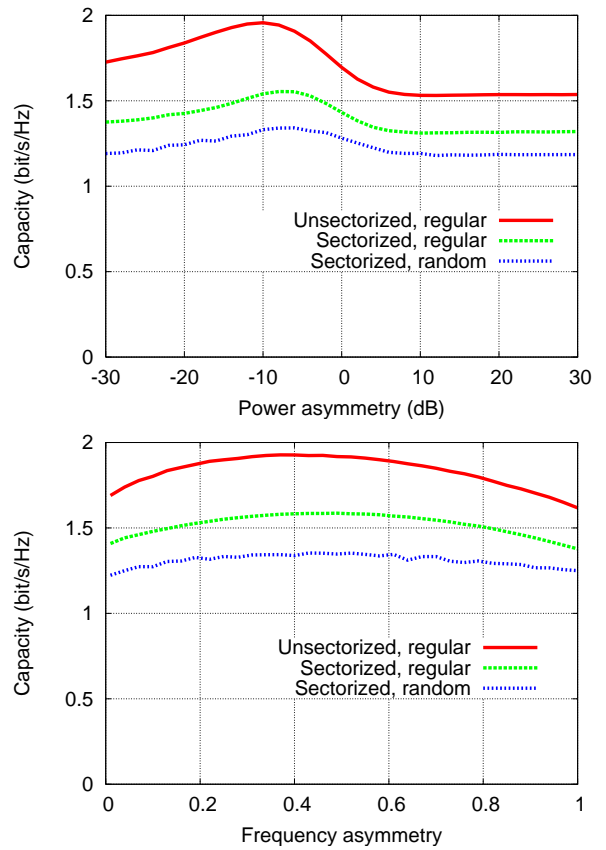

Fig. 5. Cell capacity in hexagonal networks under soft reuse (top) and fractional reuse (bottom).

the limiting case $\xi \rightarrow 0$ corresponds to the hard reuse scheme with reuse factor $1 / 3$ (the cell region $\mathcal{C}_{1}^{\star}$ shrinks so that all users are considered as far) while the case $\xi \rightarrow 1$ corresponds to the full reuse scheme (the cell region $\mathcal{C}_{2}^{\star}$ shrinks all users are considered as near). We observe that the maximum cell capacity under fractional reuse coincides with that achieved under soft reuse. This suggests that no significant capacity gains should be expected beyond those provided by soft reuse.

\section{F. Impact of radio environment}

To conclude the analysis of capacity gains, we study the impact of various parameters that characterize the radio environment: path loss exponent, angular path loss factor, thermal noise. The impact of the latter depends on the network density: it is negligible in dense networks (urban environment) and significant in sparse networks (rural environment). We measure the thermal noise power through the distance at which it is equal to the received power of the pilot signal, in the beam 
axis of any given antenna. We refer to as rural, suburban and urban, environments where this distance is equal to the cell radius, twice as long as the cell radius, and infinity, respectively. Note that the scenario without thermal noise considered until now corresponds to the urban environment.

Figure 6 below shows the respective impact of each factor on the capacity of sectorized, regular hexagonal networks under soft frequency reuse. In each case, one parameter of the reference scenario ( $\alpha=3.5, h_{0}=9 \mathrm{~dB}$, urban environment) is modified.

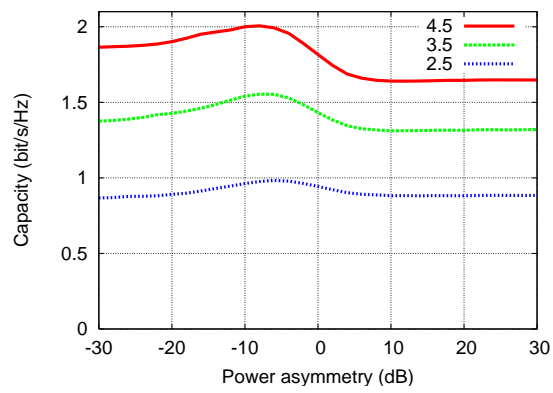

(a) Impact of path loss exponent $\alpha$

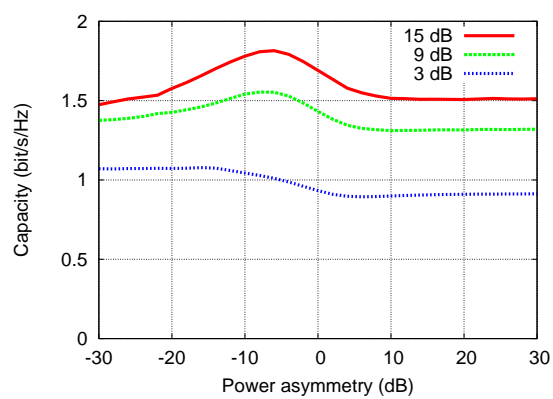

(b) Impact of angular path loss factor $h_{0}$

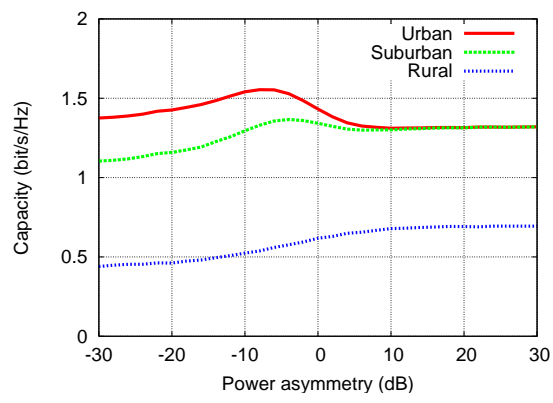

(c) Impact of network density

Fig. 6. Impact of radio environment on cell capacity under soft frequency reuse.

We observe that the capacity gain of soft reuse increases with the path loss exponent: it grows from $8 \%$ for $\alpha=2.5$ to $18 \%$ for $\alpha=4.5$. The optimal factor of power asymmetry is approximately constant, around $-5 \mathrm{~dB}$. The impact of the angular path loss factor on the capacity gain is limited; for large beams $\left(h_{0}=3 \mathrm{~dB}\right)$, where inter-sector interference is high, the optimal soft reuse scheme turns out to coincide with the hard reuse scheme with reuse factor $1 / 3$ (case $\kappa \rightarrow-\infty$ ).

Finally, Table $\Pi$ below gives the capacity gains in the urban, suburban and rural environment under soft reuse with power asymmetry $\kappa=-5 \mathrm{~dB}$ and under hard reuse with reuse factor $1 / 3$. As expected, the capacity gains are most significant in urban environments, where inter-cell interference is maximum. In all cases, the hard reuse scheme with reuse factor $1 / 3$ decreases (or at best doesn't change) the cell capacity. In rural environment, the hard reuse scheme with reuse factor $2 / 3$ turns out to be optimal (case $\kappa \rightarrow+\infty$ ).

TABLE II

IMPACT OF NETWORK DENSITY ON CAPACITY GAINS.

\begin{tabular}{|c|c|c|}
\hline Network density & Hard reuse & Soft reuse \\
\hline Urban & $0 \%$ & $+12 \%$ \\
Suburban & $-16 \%$ & $+5 \%$ \\
Rural & $-31 \%$ & $+8 \%$ \\
\hline
\end{tabular}

\section{CONCLUSION}

We have analysed the potential capacity gains of some frequency reuse schemes in various network configurations. The approach accounts for the random nature of traffic: flows of random size are generated at random times by randomly located users. In this context, we refer to the capacity of any given cell as the maximum traffic intensity it can sustain.

Our results show maximum capacity gains of $40 \%$ to $50 \%$ for linear networks and 5\% to $20 \%$ for hexagonal networks, compared to the basic full reuse scheme. In most cases, these gains cannot be achieved by hard reuse but requires either soft or fractional reuse. Surprisingly, the two latter schemes achieve the same maximum capacity gains, which suggests that no significant gains should be expected beyond those provided by soft reuse.

\section{REFERENCES}

[1] 3GPP, TSG-RAN R1-061374, "Downlink inter-cell interference coordination/avoidance of frequency reuse," Ericsson, 2006.

[2] 3GPP, TSG-RAN R1-050841, "Further analysis of soft frequency reuse scheme," Huawei, Sept. 2005.

[3] 3GPP, TSG-RAN R1-050738, "Interference mitigation - considerations and results on frequency reuse," Siemens, Sept. 2005.

[4] J. Kim, H. Son, and L. Sanghoon, "Frequency reuse power allocation for broadband cellular networks," IEICE Transactions on Communications, vol. E80-B, no. 2, Feb. 2006.

[5] A. L. Stolyar and H. Viswanathan, "Self-organizing dynamic fractional frequency reuse in OFDMA systems," in Proceedings of IEEE INFOCOM, 2008

[6] Y. Xang, J. Luo, and C. Hartmann, "Inter-cell interference mitigation through flexible resource reuse in OFDMA based communication networks," in Proceedings of European Wireless, 2007.

[7] P. Viswanath, D. N. Tse, and R. Laroia, "Opportunistic beamforming using dumb antennas," IEEE Transactions on Information Theory, vol. 48, no. 6, pp. 1277-1294, June 2002.

[8] T. Bonald, S. Borst, and A. Proutière, "Inter-cell scheduling in wireless data networks," in Proc. of European Wireless Conference, 2005.

[9] J. G. Dai, "On positive harris recurrence of multiclass queueing networks: a unified approach via fluid limit models," Annals of Applied Probability, vol. 5, pp. 49-77, 1995.

[10] P. Bender, P. Black, M. Grob, R. Padovani, N. Sindhushayana, and A. Viterbi, "CDMA/HDR: A bandwidth-efficient high-speed wireless data service for nomadic users," IEEE Commun. Magazine, pp. 70-77, July 2000.

[11] 3GPP, "Spatial channel model AHG (combined ad-hoc from 3GPP \& 3GPP2), Spatial channel model text description," SC:-062, 2002. 